\title{
Design and Modeling of Spiral Circular Magnetic Probe for Near Field Measurements in CST Microwave Studio
}

\author{
Abdulghafor A. Abdulhameed \\ Department of Electronics and \\ Information Technology \\ Faculty of Electrical Engineering \\ University of West Bohemia \\ Pilsen, Czech Republic \\ abdulhme@fel.zcu.cz
}

\author{
Zdeněk Kubík \\ Department of Electronics and \\ Information Technology \\ Faculty of Electrical Engineering \\ University of West Bohemia \\ Pilsen, Czech Republic \\ zdekubik@fel.zcu.cz
}

\begin{abstract}
This paper presents the designing, modeling, and simulation of the spiral circular magnetic field probe for near field measurements. Two configurations of this probe have been investigated using both reference boards - conventional and microstrip. Furthermore, this probe has a compact size of $(4 \times 5)$ $\mathrm{mm}^{2}$ printed on an FR-4 substrate of $1.6 \mathrm{~mm}$ height. This probe's operation frequency covers a range from $1 \mathrm{MHz}$ to $1 \mathrm{GHz}$, making it suitable to measure the electromagnetic interference in the near field region over PCB working at electromagnetic compatibility applications.
\end{abstract}

Keywords-DUT, EMC Application, Magnetic Field, Microstrip Probe

\section{INTRODUCTION}

Recently, electronic devices are being smaller and getting integrated into small packages. Therefore, the spectrum will be crowded with all electromagnetic waves since each of these integrated circuits radiates its signals. The interference of these signals is called electromagnetic interference (EMI) [1]. There are several methods to avoid EMI and allow electronic devices to work together without affecting each other, called Electromagnetic Compatibility (EMC) [2]. The EMI can occur either in the far-field or in the near field, depending on radiation source power. If the source is significant, the best way to measure the interference is to use an antenna like a sensor to estimate the electrical field $[3,4]$. Moreover, probes are used to measure interference when the source is small such as measuring the magnetic field surrounding the printed circuit board (PCB). In general, the probes are classified into two types, electrical probes, and magnetic probes. The magnetic probes need a small area (reference board) with the device under test (DUT)[5]. The ordinary magnetic probes can be easily fabricated by hand with a circular or elliptical loop. Therefore, they are not suitable for high-resolution measurements due to low accuracy[6]. On the other hand, the microstrip magnetic probes have the advantages of low cost, low profile, and easy fabrication, which make them suitable for magnetic field measurement with high resolution [7]. Many studies of design magnetic probes based on PCB are proposed. Authors in [8] present a low-cost ultra-wideband near field probe based on FR-4 substrate. [9] suggests a novel circular loop to work as a magnetic probe in the near field measurements. A set of quasi-periodic notches are engraved in the transmission line to get high effectively of eliminating the unwanted resonance of the circular loop. A compact ultrawideband magnetic probe is designed and simulated in [10]. The structure of the probe consists of a four-layer PCB of Rogers substrate.
This work is dedicated to designing a microstrip magnetic probe to estimate the induced magnetic field over PCB. The paper is organized as follows; Section II describes the design of the reference board and the design of the magnetic probe. The magnetic probe results for different frequency bands are shown in Section III, while section IV presents a brief Conclusion.

\section{DESIGNING AND SIMULATION OF MAGNETIC PROBE}

\section{A. Reference Board Design}

To model the proposed probe, we need a reference board that gives us reference values of the magnetic field to compare with the presented probe results. This reference board is necessary to detect the magnetic field. A conventional type of reference board consists of a cylindrical line of diameter $1.2 \mathrm{~mm}$ above the ground plane $\mathrm{h}=2.05 \mathrm{~mm}$ and terminated with $50 \mathrm{ohm}$. The air separates between the cylindrical line and the ground plane; this type is well known due to the easy calculation of the electromagnetic fields around the structure using the boundary image equations [11]. However, this article uses another kind of reference board called microstrip reference board, which consists of a microstrip line of width $w_{f}$ engraved in the top layer of the double-sided PCB for the purpose of accuracy. This board can be quickly and accurately fabricated at a low cost. Both conventional and microstrip reference boards are presented in Fig.1. The width of the microstrip line $w_{f}$ that provides $50 \mathrm{ohm}$ impedance matching can be calculated from equation (1):

$$
Z_{0}=\frac{87}{\sqrt{\varepsilon_{r}+1.41}} \ln \left(\frac{5.98 \times h}{0.8 \times w_{f}}\right),
$$

where $h$ is the height of the substrate and $\varepsilon_{r}$ is the relative permittivity.

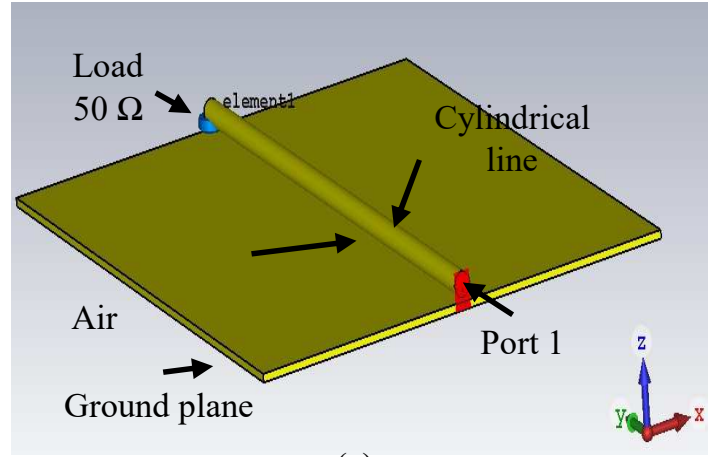

(a) 


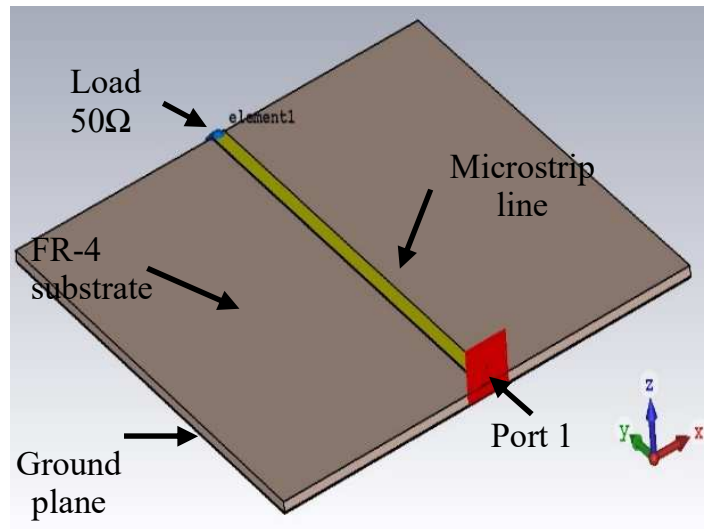

(b)

Fig.1: The conventional and proposed reference plane for the test a) conventional reference plane, and b) microstrip reference board

Fig.2 depicted a magnetic field's tangent component for both conventional and microstrip reference boards at $100 \mathrm{MHz}$ (low frequencies) and $1 \mathrm{GHz}$ (high frequencies), respectively. It can be seen that the result achieved from the microstrip reference plane has higher accuracy detection than the result achieved from the conventional reference plane. And it is apparent in the two minimum points of the red curve compared to the two minimum points of the blue curve. It can be concluded that the microstrip reference board has better performance than a conventional reference board because both the microstrip reference board and the device under the test are based on PCB technology, and they almost have the same substrate.

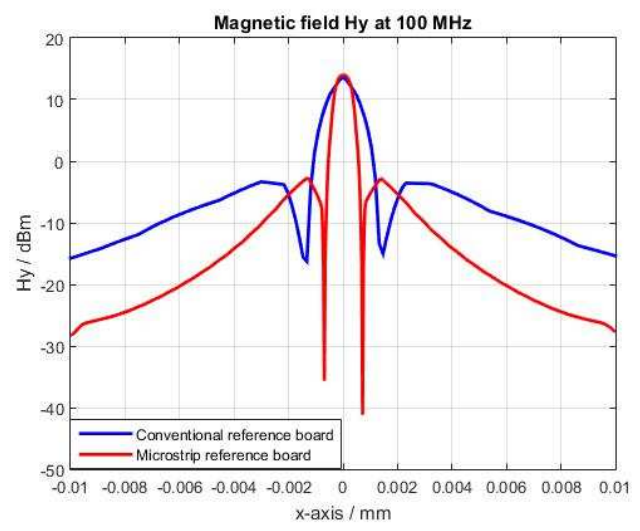

(a)

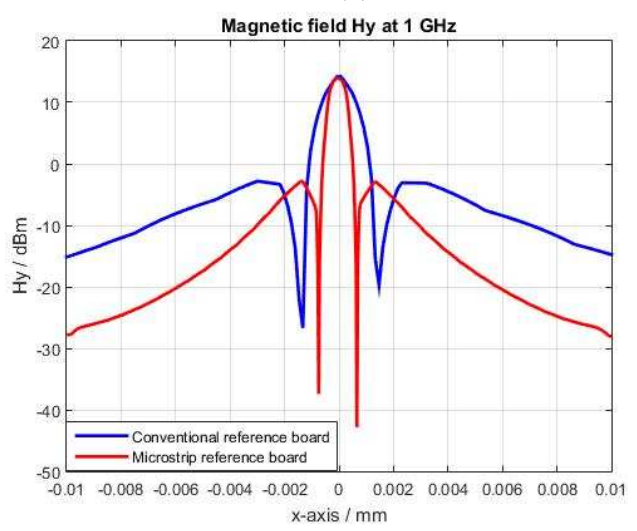

(b)

Fig.2: The magnetic field result of conventional and microstrip reference board a) $100 \mathrm{MHz}$, and b) $1 \mathrm{GHz}$.

\section{B. Design of the Probe}

The next step is to evaluate the induced magnetic field by the proposed probe, then compares it with the estimated same magnetic field by the reference board. Unfortunately, the CST Microwave studio doesn't evaluate the induced magnetic field by the probe. Instead of that, we can determine the induced voltage across the probes' side at each position. Therefore, sweeping the proposed probe from the negative $\mathrm{x}$-axis to the positive $\mathrm{x}$-axis will give us the induced voltage curve with distance. In the design procedure, we have many geometrical options to configure the proposed microstrip probe. Microstrip spiral circular probe has been designed and simulated in CST Microwave studio with two configurations [12]. Fig.3 shows the geometrical shape of the proposed probe.

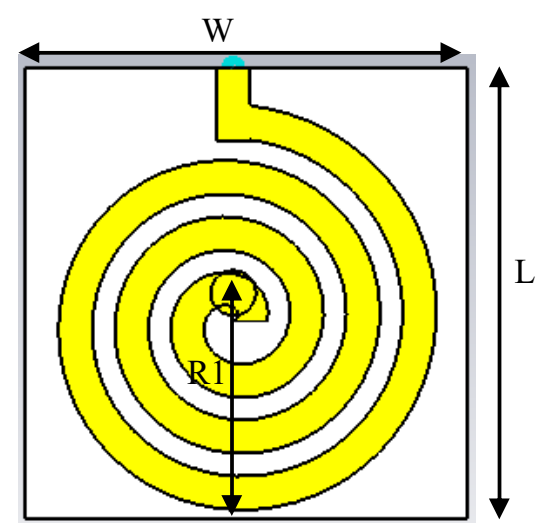

Fig.3: The geometrical shape of the printed circular probe

The dimensions of the probe are illustrated in Table I.

TABLE I. THE OPTIMUM DIMENSION OF THE SPIRAL CIRCULAR PROBE

\begin{tabular}{|c|c|}
\hline Parameter & Value (mm) \\
\hline Hight (h) & 1.6 \\
\hline L & 5 \\
\hline Trace width & 0.3 \\
\hline Gap & 0.2 \\
\hline Width (W) & 4 \\
\hline Radius (R1) & 2 \\
\hline Number of turns & 3 \\
\hline
\end{tabular}

The antenna factor is inversely proportional to the crosssection area, as shown in equation (2). Meanwhile, this area can be calculated using the circular area formula from equation (3) since the probe's shape is spiral circular.

$$
\begin{aligned}
& A_{f}=\frac{1}{\omega \mu S}, \\
& S=2 \pi r^{2} .
\end{aligned}
$$

where $\mu$ is the material's permeability, $\omega$ represents the angular frequency in rad/sec, and $r$ is the radius of the loop.

The antenna factor for this shape is evaluated and implemented in MATLAB Software. Fig.4 shows the Matlab calculation of the antenna factor. 


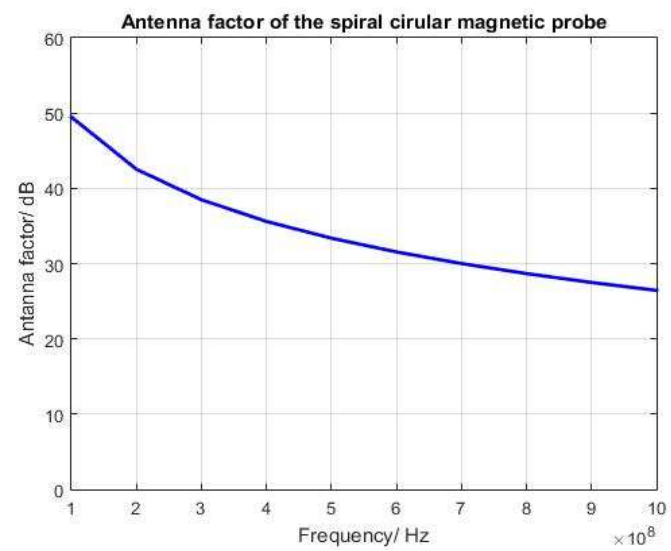

Fig.4: Antenna factor vs. Frequency (MATLAB).

\section{THE SIMULATION RESULTS}

Two configurations of microstrip spiral circular magnetic probes have been investigated, one face three-loop probe (1F3L) and two faces three-loop probe (2F3L). Fig.5 presents these two configurations. The induced magnetic field probe results for both structures at different frequencies are shown in Fig.6. at low frequency $(100 \mathrm{MHz})$ it can be seen that both configurations can detect the magnetic field even though (1F3L) has higher accuracy rather than $(2 \mathrm{~F} 3 \mathrm{~L})$. i.e., the two minimum points of the black-dashed curve have better performance than the two minimum points of the blue-dashed curve compared to the reference field (red curve). This advantage of $(1 \mathrm{~F} 3 \mathrm{~L})$ comes from the fact that the estimation area for the $(1 \mathrm{~F} 3 \mathrm{~L})$ probe is more selective than the other probe $(2 \mathrm{~F} 3 \mathrm{~L})$. As the frequency increases from $100 \mathrm{MHz}$, the (2F3L) probe's accuracy decreases while the $(1 \mathrm{~F} 3 \mathrm{~L})$ probe still has the same precision, which is obviously at $300 \mathrm{MHz}$ $500 \mathrm{MHz}$ in Fig.6b and Fig.6c, respectively. At $1 \mathrm{GHz}$, the (2F3L) probe can no longer detect the magnetic field because it doesn't have any minimum points anymore. In contrast, the (1F3L) probe can detect the magnetic field since it has two minimum points of $-30 \mathrm{~dB}$ and $-20 \mathrm{~dB}$ as demonistrated in Fig.6d.

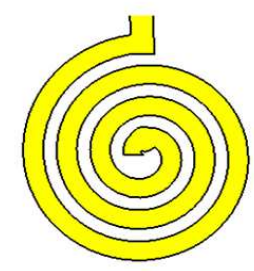

(a)

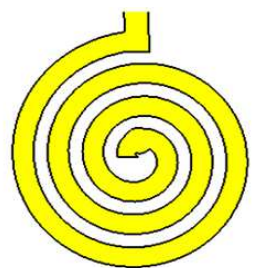

(c)

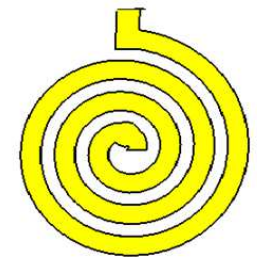

(b)

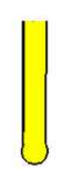

(d)
Fig.5: Two faces $(2 \mathrm{~F})$ probe versus one face (1F). a) front view $(2 \mathrm{~F})$, b) back view $(2 \mathrm{~F}), \mathrm{c})$ front view $(1 \mathrm{~F})$ and d) back view (1F).

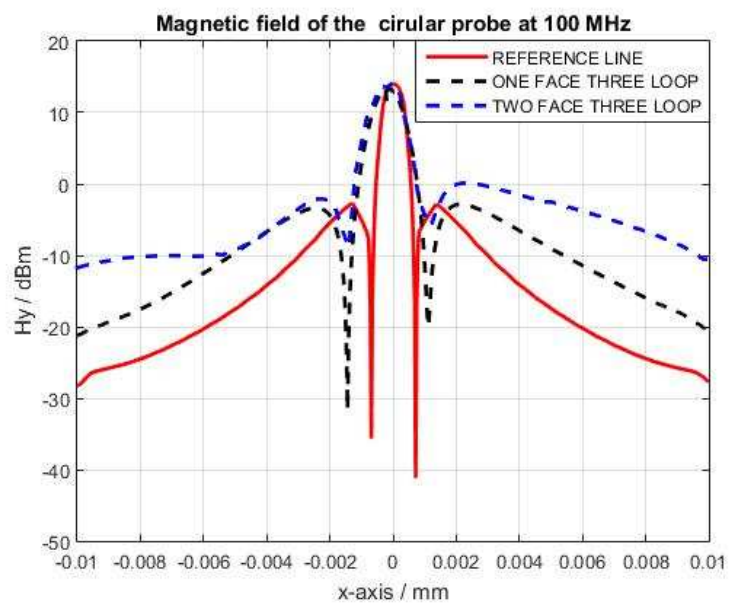

(a)

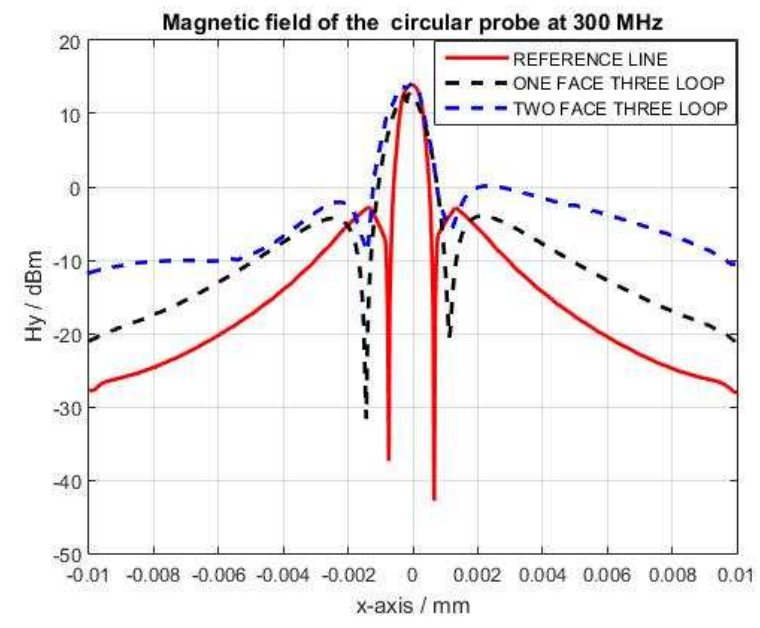

(b)

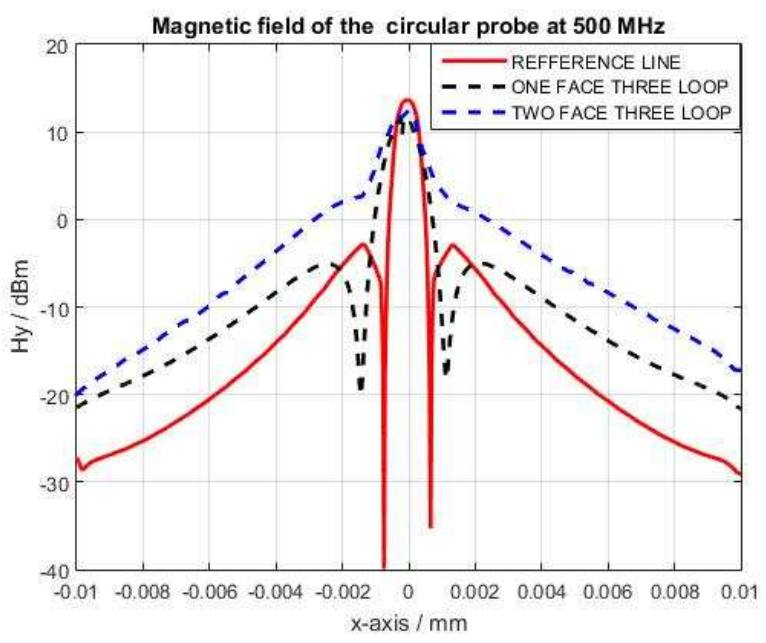

(c) 


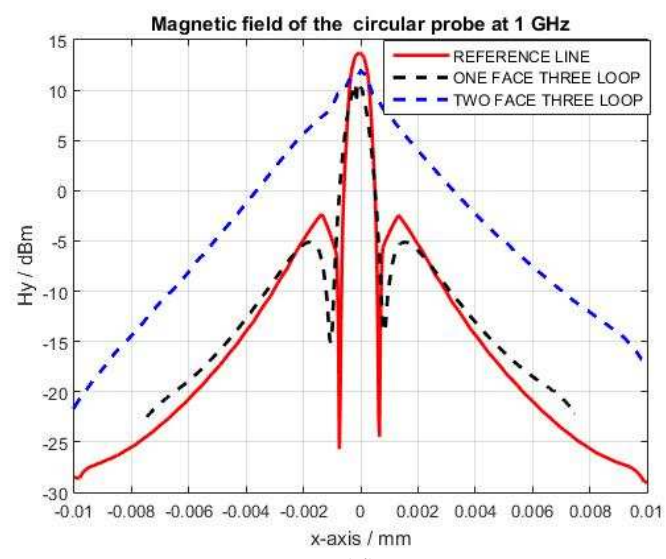

(d)

Fig.6: Magnetic field estimation for both probe configurations at low and high frequencies, a) low frequency $(100 \mathrm{MHz})$, b) $300 \mathrm{MHz}$ c) $500 \mathrm{MHz}$, and d) high frequency $(1 \mathrm{GHz})$.

Fig. 7 shows the S-parameters of the proposed antenna for both configurations $(2 \mathrm{~F} 3 \mathrm{~L}$ and $1 \mathrm{~F} 3 \mathrm{~L})$. Since the operation bandwidth determined by reflection coefficient below the -3 $\mathrm{dB}$, it's clear that the $1 \mathrm{~F} 3 \mathrm{~L}$ configuration covers the whole band up to $1 \mathrm{GHz}$, while the $2 \mathrm{~F} 3 \mathrm{~L}$ configuration is lossing his accuracy after $0.5 \mathrm{GHz}$ (the reflection is higher than $-3 \mathrm{~dB}$ ) as shown in blue dash curve.

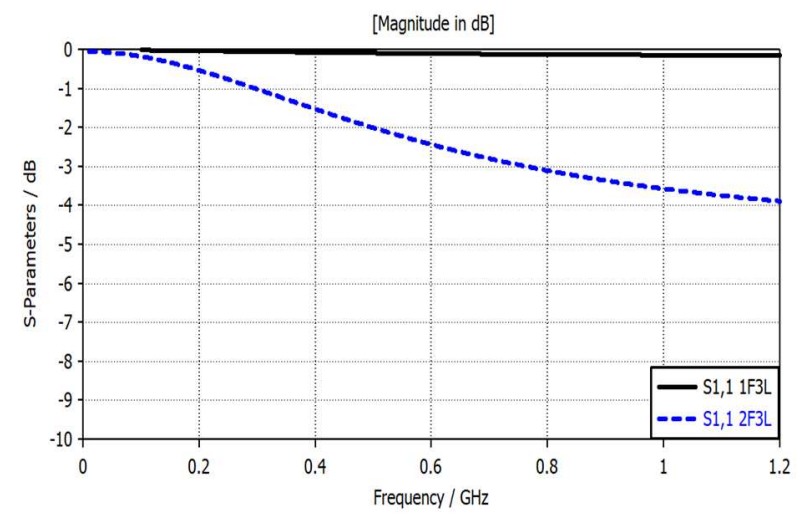

Fig.7: S11-parameters of the proposed probes (1F3L and 2F3L).

One of the key parameters of magnetic probe design is the isolation, i.e., the ability of the magnetic probe to suppress the unwanted electrical field. The S12-parameter represents the magnetic field and the electrical field when the direction of the probe parallel and perpendicular to the microstrip line of the reference board test respectively. The isolation of two configurations is about $12 \mathrm{~dB}$ as shown in Fig 8.

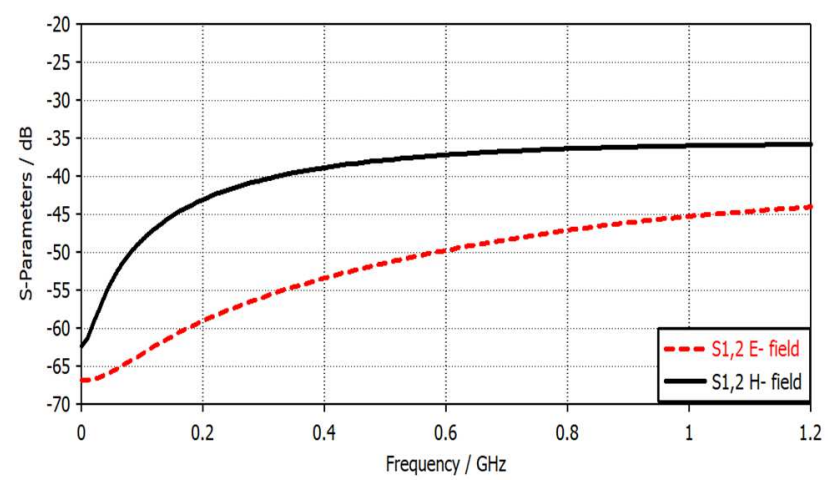

Fig.8: S12-parameters of the proposed probes (1F3L).

\section{CONCULSION}

Two configurations of a spiral circular magnetic probe (two faces three-loops (2F3L) and one face three-loop (1F3L)) are designed and simulated to serve EMI measurements. The microstrip board is used as a reference board with features closed to the DUT to validate the results collected from the proposed probe, used this new reference plane due to low cost, low profile, and ease to fabricate. The (1F3L) probe has more selective than (1F3L); therefore, it has better performance than $(2 \mathrm{~F} 3 \mathrm{~L})$. In conclusion, the $(1 \mathrm{~F} 3 \mathrm{~L})$ probe covers the frequency band from $1 \mathrm{MHz}$ to $1 \mathrm{GHz}$ effectively to estimate the magnetic field over PCB. It is worth mentioning that decreasing the number of loops has a direct impact on broadening the operation bandwidth of the probe due to decreasing the parasitic capacitor.

\section{ACKNOWLEDGMENT}

This research has been supported by the Ministry of Education, Youth and Sports of the Czech Republic under the project OP VVV Electrical Engineering Technologies with High-Level of Embedded Intelligence CZ.02.1.01/0.0/0.0/18_069/0009855 and by the project SGS2021-005: Research, development and implementation of modern electronic and information.

\section{REFERENCES}

[1] Zdeněk, K., Denys, N., Pavel, K., Jiř́, S., \& Miroslav, H. (2014). Optimization of electrical properties of parallel plate antenna for EMC testing. Journal of Computational and Applied Mathematics, 270, 283 293

[2] Wang, X., Chen, X., Shi, X., Bai, Y., Wei, F., \& Li, P. (2009, September). EMC design for an anti-jamming smart antenna system. In 2009 5th Asia-Pacific Conference on Environmental Electromagnetics (pp. 196-199). IEEE.

[3] Ahirwar, S. D., Ramakrishna, D., \& Pandharipande, V. M. (2020). Design and Development of a Broadband Planar Dipole Antenna. In Advances in Decision Sciences, Image Processing, Security and Computer Vision (pp. 185-193). Springer, Cham.

[4] Alnahwi, F. M., et al. "Mutual coupling reduction of a dual-band $2 \times 1$ MIMO antenna using two pairs of $\lambda / 4$ slots for WLAN/WiMAX applications." (2018): 10-5.

[5] Funato, H., \& Suga, T. (2006, March). Magnetic near-field probe for $\mathrm{GHz}$ band and spatial resolution improvement technique. In 2006 17th International Zurich Symposium on Electromagnetic Compatibility (pp. 284-287). IEEE.

[6] Baudry, David, A. Louis, and B. Mazari. "Characterization of the openended coaxial probe used for near-field measurements in EMC applications." Progress In Electromagnetics Research 60 (2006): 311333.

[7] Balanis, C. A. (2016). Antenna theory: analysis and design. John Wiley \& sons.

[8] Namahoot, A., Akkaraekthalin, P., \& Chalermwisutkul, S. (2019). Design of a low-cost 1-20 GHz magnetic near-field probe with FR-4 printed circuit board. International Journal of RF and Microwave Computer-Aided Engineering, 29(12), e21958.

[9] S. Lin, S. Yen, W. Chen and P. Cheng, "Printed magnetic field probe with enhanced performances," 2009 Asia Pacific Microwave Conference, Singapore, 2009, pp. 649-652, doi: 10.1109/APMC.2009.5384134.

[10] Z. Yan, J. Wang, W. Zhang, Y. Wang and J. Fan, "A Simple Miniature Ultrawideband Magnetic Field Probe Design for Magnetic Near-Field Measurements," in IEEE Transactions on Antennas and Propagation, vol. 64, no. 12, pp. 5459-5465, Dec. 2016, doi: 10.1109/TAP.2016.2606556.

[11] Sivaraman, N. (2017). Design of magnetic probes for near field measurements and the development of algorithms for the prediction of EMC (Doctoral dissertation, Université Grenoble Alpes).

[12] CST(2014) Computer Simulation Technology Based on FIT Method 